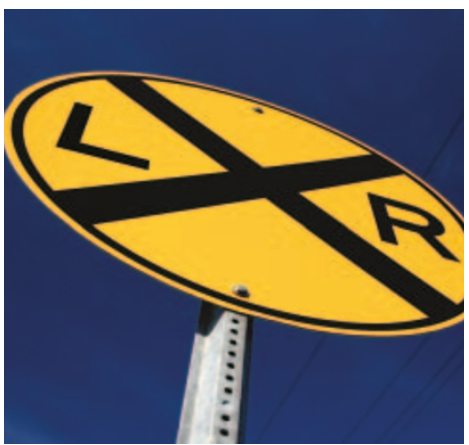

\title{
At the junction between L-R patterning and somitogenesis
}

Left-right $(\mathrm{L}-\mathrm{R})$ asymmetry is required for normal development, but how can symmetry arise in an embryo with L-R polarity? Three groups independently show that symmetry in the somites - which give rise to the skeleton and the muscles - is established by the action of retinoic acid. A fourth group shows that very early in L-R patterning, retinoic acid and Sonic Hedgehog, released in response to fibroblast growth factor (FGF) signalling and encased in membrane-bound vesicles, are transported by nodal flow to establish the initial asymmetry.

Retinoic acid has a newly discovered function - to ensure that somitogenesis occurs synchronously on the left and on the right sides of the embryo. Genetic and embryological experiments in the mouse, chick and zebrafish show that when retinoic acid is absent (either because an antagonist has been administered to the embryo or because the enzyme that synthesizes retinoic acid has been removed genetically), new somites develop asynchronously. Changes in the expression patterns of genes involved in the well-defined process of somitogenesis revealed that Lunatic fringe and other so-called cycling genes (such as deltaC or her1) are asymmetrically deregulated. This asynchrony, which is indicative of a deregulation of the 'clock' - a mechanism that sets the periodicity with which new somites form - is not random: supernumerary somites form on the left. This bias disappears when 'situs' is randomized in the embryo. In the chick, it can be done by inducing Sonic Hedgehog activity on the right side, which does not itself affect somitogenesis, in mouse by using the $i v^{-/-}$mutants and in zebrafish by inhibiting $\mathrm{H}^{+} / \mathrm{K}^{+}$ATPase activity or by preventing left-right dynein expression (Kawakami and Raya et al. show that both of these function very early in L-R asymmetrical patterning).

Early in embryogenesis, Notch signalling is upregulated on the left, which in turn activates Nodal signalling and the rest of the L-R patterning pathway. Downstream of Notch lies Lunatic fringe, a key cycling gene that coordinates somite formation and for which expression becomes asymmetrical in response to the asymmetrical Notch activation. What these new data show is that retinoic acid compensates for this initial asymmetry to allow for symmetrical somitogenesis in the zebrafish, chick and mouse. Interestingly, because Amphioxus somitogenesis is asymmetrical, this function of retinoic acid is probably vertebrate-specific.

A related report shifts the focus away from somitogenesis to the earliest events in the establishment of L-R asymmetry. It is well established that cilia-mediated leftward flow around the embryonic node is crucial for the breaking of L-R symmetry in mammals. Kawakami and Raya et al. provide the first experimental evidence for the 'nodal flow hypothesis' in zebrafish. Tanaka et al. now show that, at least in mouse embryos, this flow transports small (0.3-5 $\mu \mathrm{m})$ membrane-bound 'nodal vesicular parcels' that contain Sonic Hedgehog and retinoic acid. Their pharmacological and molecular genetic experiments indicate that these vesicles are released from the surface of the node and its dynamically protruding microvilli in response to FGF signalling.

With so much new data for the key developmental models we should be able to look forward to some interesting evo-devo studies in the near future.

\section{(4) References and links ORIGINAL RESEARCH PAPERS Vermot, J. \&} Gallego-Llamas, J. G. et al. Retinoic acid controls the bilateral symmetry of somite formation in the mouse embryo. Science 308, 563-566 (2005) | Kawakami, Y. \& Raya, A. et al. Retinoic acid signalling links left-right asymmetric patterning and bilaterally symmetric somitogenesis in the zebrafish embryo. Nature 435, 165-171 (2005) | Tanaka, Y. et al. FGF-induced vesicular release of Sonic hedgehog and retinoic acid in leftward nodal flow is critical for left-right determination. Nature 435, 172-177 (2005) | Vermot, J. and Pourquié, O. Retinoic acid coordinates somitogenesis and leftright patterning in vertebrate embryos. Nature 435, 215-220 (2005)

FURTHER READING Okada, Y. et al. Mechanism of nodal flow: a conserved symmetry breaking event in left-right axis determination. Cell 121, 633-644 (2005) | Hamada, H. et al. Establishment of vertebrate left-right asymmetry. Nature Rev. Genet. 3, 103-113 (2002) | Saga Y. \& Takeda, H. The making of the somite: molecular events in vertebrate somitogenesis. Nature Rev. Genet. 2, 835-845 (2001) | Hornstein, E. \& Tabin, C. J. Asymmetrical threat averted. Nature 435, 155156 (2005) 